\title{
两种雌雄同株榕树榕果内小蜂空间分布格局
}

\author{
王振吉 ${ }^{1,2}$, 李国昌 ${ }^{3}$, 彭艳琼 ${ }^{1}$, 杨大荣 ${ }^{1, *}$ \\ (1. 中国科学院西双版纳热带植物园, 云南 昆明 650223 ; 2. 楚雄师范学院化学与生命科学系, 云南 楚雄 675000 ; \\ 3. 楚雄市林业局, 云南 楚雄 675000)
}

\begin{abstract}
摘要: 在雌雄同株榕树榕果内, 除传粉榕小蜂外, 还寄生着很多种类的非传粉榕小蜂。为弄清传粉和非传粉 榕小蜂在榕果内的空间分布格局, 以西双版纳地区的垂叶榕和高榕及与之相关的传粉和非传粉榕小蜂为研究材料, 比较了不同种类小蜂所在瘃花花梗长度差异。结果表明: (1) 在高榕中, Eupristina sp.所在瘞花花梗平均长度最长, 传粉榕小蜂 Eupristina altissima 所在瘦花花梗长度次之。Micranisa ralianga、Sycobia sp.和 Sycoscapter sp. 2 所在 瘦花花梗长度无显著差异。Sycobia sp.、M.ralianga 以及 Sycoscapter sp. 2 所在痔花花梗长度范围要窄于传粉榕小 蜂所在瘦花花梗长度范围。这都说明这三种果外产卵非传粉榕小蜂与传粉榕在空间生态位上存在部分的分离。(2) 在垂叶榕中, Eupristina koningsbergeri 可以分布在从最外层到内层的㾉花, 而大多数 Walkerella sp. 都集中在靠近果 壁的瑤花内，表明两者也存在部分空间生态位的分离。
\end{abstract}

关键词: 榕小蜂; 空间分布; 瘞花; 花梗

中图分类号: Q969. 54; Q948.122.1＼cjkstart文献标志码：A＼cjkstart文章编号：0254-5853-(2012)02-0182-05

\section{Spatial distribution of fig wasps in syconia of two monoecious Ficus sp.}

\author{
WANG Zhen-Ji ${ }^{1,2}$, LI Guo-Chang ${ }^{3}$, PENG Yan-Qiong ${ }^{1}$, YANG Da-Rong ${ }^{1, *}$ \\ (1. XishuangbannaTropical Botanical Garden, Chinese Academy of Science, Kunming 650223, China; \\ 2. Department of Chemistry and Life Science, Chuxiong Normal University, Chuxiong 675000, China; \\ 3. Forestry Bureau of Chuxiong City, Chuxiong 675000, China)
}

\begin{abstract}
In addition to pollinator fig wasps, there are several non-pollinating fig wasps associated with monoecious Ficus sp. In order to understand how pollinator fig wasps and non-pollinating fig wasps are distributed across the same syconium, the spatial distribution of fig wasps associated with Ficus altissima and F. benjamina were compared using the pedicle lengths of galls containing each species. The results indicate that in Ficus altissima, the average pedicel length of galls containing Eupristina sp. is longer than that containing E. altissima. Average pedicel length of galls containing Sycobia sp., Micranisa ralianga and Sycoscapter sp. two did not show significant difference. The range of pedicel lengths of galls containing Sycobia sp., M. ralianga or Sycoscapter sp. two is narrower than that of galls containing E. altissima, indicating these non-pollinating fig wasps and pollinator have partially separated spatial niches. In F. benjamina, E. koningsbergeri was distributed in galls from the outer layer to inner layer, while most Walkerella sp. were found in outer layer galls, indicating E. koningsbergeri and Walkerella sp. have partially separated spatial niches.
\end{abstract}

Key words: Fig wasp; Spatial distribution; Gall; Pedicel

榕树与榕小蜂互利共生体系被认为是动植物 间互作关系最特化、最具代表性的互利共生系统之 一(Janzen, 1979; Wiebes, 1979; Herre et al, 1999)。在 榕-蜂共生体系中, 除传粉榕小蜂外, 还生活着许多
非传粉榕小蜂(Hawkins \& Compton, 1992; West \& Herre, 1994; Kerdelhué \& Rasplus, 1996a; Jousselin et al, 2001)。非传粉榕小蜂一直以来都被认为对互 利共生系统具有负面的影响(Kerdelhué \& Rasplus,

收稿日期：2011-05-08; 接受日期：2011-12-13

基金项目: 国家自然科学基金(30970403, 30970439); 云南省应用基础基金(2011FZ186)

*通信作者(Corresponding author), E-mail: yangdr@xtbg.ac.cn

第一作者简介: 王振吉 (1983-), 男, 博士, 楚雄师范学院教师, 主要从事进化生态学研究 
1996a)。传粉和非传粉榕小蜂后代在榕果内有不同 的空间分布格局(Kerdelhué \& Rasplus, 1996b; Zhai et al, 2008), 这可能降低非传粉榕小蜂对传粉小蜂 的危害性。

在雌雄异株榕树中, 雌树榕果中小花的花柱较 长, 不适合传粉榕小蜂产卵 (Nefdt \& Compton, 1996), 雄树榕果的雌花花柱较短, 这些雌花被传粉 榕小蜂或非传粉榕小蜂所寄生, 所以尽管雄果中有 雌花和雄花, 但是在功能上, 它们是雄性的, 只能 产生花粉和小蜂(Kjellberg et al, 1987); 而在雌雄同 株榕树中, 每个榕果既会产生种子又会产生榕小蜂 (Yang et al, 2001)。在雌雄同株榕树中, 由于花梗长 度差异, 榕果的雌花分数层排列, 但是几乎所有雌 花的柱头都呈现在同一个平面上, 这是由于花柱和 花梗长度的变异所致(Ganeshaiah et al, 1999)。靠近 果腔的为内层, 靠近果壁的为外层, 在外层和内层 中间的是中层 (Kerdelhué \& Rasplus，1996b; Kerdelhué et al, 2000)。在雌雄同株榕果中, 传粉榕 小蜂偏爱于把卵产在靠近果腔花柱较短的小花中, 而位于靠近果壁花柱较长的花产生种子(Nefdt \& Compton, 1996; Anstett, 2001; Jousselin et al, 2001)。 Kerdelhué \& Rasplus(1996a)研究了非洲的非传粉榕 小蜂对聚果榕亚属榕树榕-蜂互利共生系统的影响, 发现进入 Ficus sur 榕果内产卵的Sycophaga silvestrii 在大多数情况下会把卵产在内层小花, 也 就是短花柱小花内。只有在繁殖䧳蜂数量较多, 对 产卵位点竞争激烈的情况下才会把卵产在外层小 花内, 但在这个层次小花上产卵的数量仍然很少。 Apocryptophagus sp. 2 和 Apocryptophagus sp. 3 也将 卵产在内层小花内。另外, 在 Ficus vallis-choudae 产卵的 Apocryptophagus sp. 4 也将卵产在内层小 花。与 Kerdelhué \& Rasplus (1996a)的研究结果不同, Zhai et al (2008)研究了西双版纳聚果榕隐头果内传 粉和非传粉榕小蜂空间分布格局, 发现除 Platyneura testacea 外, 其他种类非传粉榕小蜂 (Platyneura mayri、Apocrypta westwoodi、Apocrypta sp.)主要利用榕果外层小花。以上两研究结果虽然 存在差异, 但都说明非传粉榕小蜂会在利用榕果内 不同层次小花时具有一定倾向性。然而，上述所有 研究都仅仅把榕果内小花分为内层、外层、中层三 个层次, 但事实上榕果内小花长度是连续型定量数 据, 而非定性数据, 仅仅分成三个层次, 必然会导 致研究结果存在一定的偏差。所以, 在研究榕果内
小蜂空间分布格局过程中，有必要将花梗的长度细 分化。

高榕 (Ficus altissima Blume) 和垂叶榕 (Ficus benjamina Linnaeus) 是西双版纳地区常见雌雄同株 榕树种类。两种榕树都属于榕属、环纹榕组, 但榕 果物候期存在明显差异。两种榕树中都寄生着多种 非传粉榕小蜂, 其中高榕中有 24 种非传粉榕小蜂 (Gu et al, 2003); 垂叶榕中有 12 种非传粉榕小蜂(Bai et al, 2008), 但两种榕树的非传粉榕小蜂种类均不 同。本研究以高榕、垂叶榕, 以及与其相关的传粉 和部分非传粉榕小蜂为材料, 研究了两种榕树榕果 内各种小蜂空间分布规律的异同，以回答如下两个 主要问题: 1)两种榕树榕果内传粉和非传粉榕小蜂 是否存在空间生态位分离; 2) 两种榕树榕果内各种 非传粉榕小蜂之间是否存在空间生态位分离。

\section{1 材料与方法}

\section{1 材料}

研究样地一西双版纳热带植物园(XTBG)位于 云南省西双版纳傣族自治洲境内。地理位置为 $\mathrm{N} 21^{\circ} 41^{\prime} \sim 22^{\circ} 36^{\prime}, \mathrm{E} 99^{\circ} 56^{\prime} \sim 101^{\circ} 25^{\prime}$, 海拔 $560 \sim 680$ $\mathrm{m}$, 地处东南亚热带北缘, 为热带季风性气候(Guan et al, 2007)。

高榕(Ficus altissima Blume)隶属桑科(Moraceae), 榕属榕亚属(Urostigma) 组 (Urostigma), 环纹榕亚 组 (Conosycea), 为高大乔木。本文涉及的与之相关 的小蜂为: 传粉榕小蜂 Eupristina altissima; 非传粉 榕小蜂 Eupristina sp.、Micranisa ralianga、Sycobia sp. 以及 Sycoscapter sp. 2。

垂叶榕 (Ficus benjamina Linnaeus)也为环纹榕 亚组的乔木。本文涉及的与之相关的小蜂为: 传粉 榕小蜂 Eupristina koningsbergeri 和非传粉榕小蜂 Walkerella sp.。

\section{2 方法}

1.2.1 小蜂所在瘞花花梗长度测量方法 2008 年, 开始在西双版纳热带植物园内分别选取 1 株高榕和 1 株垂叶榕。当两种榕树结果后，分别观察各种小 蜂在两种榕树隐头果的产卵顺序和产卵情况, 并标 记各种小蜂产卵比较集中的区域。当榕果发育至雄 花期, 小蜂尚未离开㾉花, 但是种类已经可以辨别 时, 在标记区域采摘高榕和垂叶榕的榕果各 10 个 带回实验室，放入用 75\%酒精内，使瘘花内小蜂死 亡, 从而避免其离开㾉花进入果腔。在实验室内测 
量并记录隐头果的直径、轴长。然后将榕果剖开, 用 铒子挑出掼花, 置于培养血中, 在 OLYMPUSSZX12 体视镜下测量㾉花花梗长度。每测完一个㾉 花后, 用铌子夹裂瘞花, 将小蜂从瘦花中用铌子挑 出, 然后鉴定其种类并做好记录。

1.2.2 数据分析方法 采用单因素方差分析比较 各种小蜂所在瘘花的花梗长度差异。其中, 方差同 质性检验采用 Levene 检验, 多重比较采用 LSD 法。 采用单样本 $\mathrm{K}-\mathrm{S}$ 检验对各种小蜂所在掼花花梗长度 进行正态性检验。所有分析都采用 SPSS16.0 软件。

\section{2 结 果}

\section{1 高榕榕果内小蜂的空间分布}

在高榕中, Eupristina sp.所在瘞花花梗平均长 度最长, 传粉榕小蜂 E. altissima 所在㾉花花梗长度 次之。M. ralianga 和 Sycoscapter sp. 2 所在瘦花花 梗长度无显著差异。虽然 Sycobia $\mathrm{sp}$. 所在瘿花花梗 长度与 M. ralianga 以及 Sycoscapter sp. 2 所在㾉花 花梗长度均存在差异, 但统计学检验不显著(表 1)。

从小蜂所利用的小花花梗长度范围来看, 传粉 榕小蜂以及另一种进入果内产卵的非传粉榕小蜂 Eupristina sp. 可以占据靠近果壁、靠近果腔以及果 壁与果腔之间的瘞花。而其他 3 种果外产卵的非传
粉榕小蜂只能占据从果壁到果腔内一定长度范围

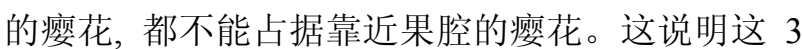
种果外产卵非传粉榕小蜂与传粉榕小蜂在空间生 态位上存在部分分离，并且这 3 种小蜂空间生态位 也并非完全重叠。其中, M. ralianga 和 Sycoscapter sp.2 所在㾉花花梗长度范围大体一致，而 Sycobia $\mathrm{sp}$. 所在瘱花花梗长度范围最窄，仅能利用从外层到 中层的小花 (表 1)。

高榕榕果内 5 种小蜂所在㾉花花梗长度都属于 正态分布(表 2)。这也表明这些小蜂的繁殖雌蜂产卵 时，可能并不能主动识别并区分具不同花梗长度的 小花; 而 3 种果外产卵非传粉榕小蜂不能较靠近果 腔的小花产卵可能是由于榕果形态以及其自身产 卵器长度所限。

\section{2 垂叶榕榕果内小蜂的空间分布}

在垂叶榕中，传粉榕小蜂 E. koningsbergeri 与 Walkerella sp.所在瘱花花梗长度平均值无显著差 异。从两种小蜂所占据瘞花花梗长度范围来看, 所 占据瑤花花梗长度范围大体一致，且分布在从外层 到内层的瘪花内，但这并不能说明两者所占据的生 态位完全重叠。Walkerella sp.所在瑤花的花梗长度 属于非正态分布，其繁殖雌蜂会将大多数卵产在靠 近果壁的小花内。

表 1 不同种类小蜂所在瑤花的花梗长度比较

Tab. 1 Comparison of pedicel length of galls containing different fig wasps

\begin{tabular}{clccc}
\hline $\begin{array}{c}\text { 榕树种类 } \\
\text { Fig species }\end{array}$ & \multicolumn{1}{c}{$\begin{array}{c}\text { 小蜂种类 } \\
\text { Fig wasp species }\end{array}$} & $\begin{array}{c}\text { 样本量 } \\
n\end{array}$ & $\begin{array}{c}\text { 花梗长度 } \\
\text { Pedicel length }(\mathrm{mm})\end{array}$ & $\begin{array}{c}\text { 花梗长度范围 } \\
\text { Range of pedicel length (mm) }\end{array}$ \\
\hline F. altissima & Eupristina altissima & 244 & $1.08 \pm 0.04^{\mathrm{a}}$ & $0.00 \sim 2.46$ \\
& Eupristina sp. & 27 & $1.36 \pm 0.12^{\mathrm{b}}$ & $0.03 \sim 2.33$ \\
& Micranisa ralianga & 25 & $0.68 \pm 0.12^{\mathrm{c}}$ & $0.00 \sim 1.87$ \\
& Sycobia sp. & 9 & $0.37 \pm 0.16^{\mathrm{c}}$ & $0.00 \sim 1.41$ \\
& Sycoscapter sp. 2 & 22 & $0.79 \pm 0.13^{\mathrm{c}}$ & $0.00 \sim 1.97$ \\
F. benjamina & Eupristina koningsbergeri & 124 & $0.90 \pm 0.06^{\mathrm{a}}$ & $0.00 \sim 2.46$ \\
& Walkerella sp. & 378 & $0.94 \pm 0.04^{\mathrm{a}}$ & $0.00 \sim 3.34$ \\
\hline
\end{tabular}

表 2 不同种类小蜂所在瘦花的花梗长度正态性检验

Tab. 2 Normal distribution test of pedicel length of galls containing different fig wasps

\begin{tabular}{clccc}
\hline $\begin{array}{c}\text { 榕树种类 } \\
\text { Fig species }\end{array}$ & \multicolumn{1}{c}{$\begin{array}{c}\text { 小蜂种类 } \\
\text { Fig wasp Species }\end{array}$} & $\begin{array}{c}\text { 样本量 } \\
n\end{array}$ & Kolmogorov-Smirnov Z & $\begin{array}{c}\text { 显著性水平 } \\
P\end{array}$ \\
\hline F. altissima & Eupristina altissima & 244 & 0.74 & 0.65 \\
& Eupristina sp. & 27 & 0.60 & 0.86 \\
& Micranisa ralianga & 25 & 0.64 & 0.80 \\
& Sycobia sp. & 9 & 0.81 & 0.52 \\
& Sycoscapter sp. 2 & 22 & 0.89 & 0.41 \\
& Eupristina koningsbergeri & 124 & 1.01 & 0.23 \\
& Walkerella sp. & 378 & 3.04 & $<0.01$ \\
\hline
\end{tabular}




\section{3 讨 论}

生态位分化假说认为, 当资源存在空间差异并 且每一物种都在其有竞争优势的位置定居时, 大量 物种可以共存(Sun et al, 2008)。每种榕树的榕果内 可以寄生多种非传粉榕小蜂, 有些榕树上寄生的非 传粉榕小蜂种类多达 30 种以上 (Compton \& Hawkins, 1992)。如此多的小蜂能够共存在同一榕果 内, 那么这些小蜂或者存在时间生态位的分化, 或 者是通过利用果内不同类型小花而达到空间生态 位的分化(Proffit et al, 2007)。在雌雄同株榕树的隐 头果内由于花梗长度的差异, 导致雌花子房分层明 显(Ganeshaiah et al, 1999)。可能正是由于这种分层 现象为多种榕小蜂共同栖息于同一榕果提供了空 间基础(Kerdelhué \& Rasplus, 1996b)。

进入榕果产卵的传粉榕小蜂的产卵器不能到 达具较长度花柱的小花子房, 它们只能在较短花柱 小花内繁殖后代(Ramirez, 1974)。本研究结果表明, 高榕和垂叶榕的传粉榕小蜂可以分布在从最外层 到最内层的瘘花中, 即它们可以利用果内不同空间 分布类型的小花资源。果外产卵的非传粉榕小蜂则 均不能利用靠近果腔的小花, 说明虽然这些非传粉 榕小蜂小蜂和传粉榕小蜂在空间生态位上存在重 叠, 但是也存在部分分离的现象。这种生态位的分 离对维持榕-蜂互利共生系统在面对寄生的情况下 维持稳定具有积极的意义。因为, 大部分果外产卵 非传粉小蜂占据外层子房可以减少对传粉小蜂的 危害, 保证了传粉小蜂的种群维持, 进而保证了榕 树的雄性功能(Zhai et al, 2008)。

在高榕中, 果外产卵的非传粉榕小蜂 Sycobia sp. 、M. ralianga 和 Sycoscapter sp.2 所在瘞花花梗长 度范围虽存在明显差异; 但三种非传粉榕小蜂所在 瘦花梗长度都属于正态分布，这表明这三种小蜂的 繁殖雌蜂可能并不会对不同花梗长度小花进行识 别, 它们选择哪些小花来产卵是一种随机行为。它 们所利用㾉花花梗长度范围差异可能是由于其产 卵器长度以及榕果不同发育历期形态共同决定的。 随着榕果的增大, 不同种类的非传粉小蜂按照不同 的时间顺序到达榕果产卵, 而它们的产卵器长度也
是不相等的, 越是后来产卵的小蜂具有越长的产卵 器(Zhai et al, 2008)。榕果果壁的增厚限制了它们在 内层子房中产卵，因而在不同的果壁厚度产卵的非 传粉小蜂也有可能把卵产到相同层次的㾉花子房 内(Zhai et al, 2008)。前期研究发现 Sycobia sp. 、M. ralianga 和 Sycoscapter sp.2 都是用产卵器刺穿榕果 果壁, 然后将卵产在小花子房或瘞花内。Sycobia sp. 在榕果发育雌花前期产卵, 虽然此时榕果较小, 果 壁和子房层较薄, 但是其产卵器可能较短, 导致其 不能利用靠近果腔的小花。Micranisa ralianga 和 Sycoscapter sp. 2 产卵器长度虽然可能较长, 但由于 它们都在间花期产卵产卵，此时榕果较大，果壁和 子房层加厚, 也导致它们不能利用靠近果腔的小 花。在垂叶榕中，果外产卵的非传粉榕小蜂 Walkerella sp.所在㾉花多接近果壁, 但由于其寄生 的瘱花及其花梗在发育过程中异常膨大，这就导致 Walkerella sp. 与 E. koningsberger 所在瘦花花梗未体 现出长度差异。

Eupristina sp. 与高榕传粉榕小蜂所占据的生态 位几乎完全重叠。Eupristina sp.虽然也属于非传粉 榕小蜂，但它与传粉榕小蜂都属于同一个属，其产 卵方式、产卵时间与传粉榕小蜂一致。重叠的生态 位必然导致 Eupristina sp. 与传粉榕小蜂之间激烈的 资源竞争。但可能是由于苍片口形态结构不仅限制 进入榕果内的传粉榕小蜂数量, 也限制了进入榕果 内 Eupristina sp.数量, 从而削弱了 Eupristina $\mathrm{sp}$.对 互利共生系统的影响。

总之, 本实验的结果表明, 在高榕和垂叶榕中, 果外产卵的非传粉榕小蜂与传粉榕小蜂, 以及这些 非传粉榕小蜂之间都存在着或多或少的空间生态 位分离，进而达到共存。而进入果内的 Eupristina sp. 与传粉榕小蜂虽然在空间生态位上重叠, 但由于苞 片口限制了进入榕果内 Eupristina sp.数量, 也导致 了 Eupristina sp. 的影响也不会导致互利共生系统的 崩溃。

致谢: 感谢西双版纳热带植物园协同进化组全 体老师和同学在实验和论文写作过程中给予的鼓 励与帮助。

\section{参考文献:}


Oikos, 95(3): 476-484.

Bai LF, Yang DR, Compton SG. 2008. A gall midge inhabiting the figs of Ficus benjamina in Xishuangbanna, south-western China[J]. Symbiosis, 45(1-3): 149-152.

Compton SG, Hawkins BA. 1992. Determinants of species richness in southern African fig wasp assemblages[J]. Oecologia, 91(1): 68-74.

Ganeshaiah KN, Kathuria P, Shaanker RU. 1999. Does optimal packing of flowers in syconia shape style length variation in monoecious figs [J]Biotropica, 31(2): 312-320.

Gu HY, Yang DR, Zhang GM, Peng YQ, Song QS. 2003. Species of fig wasps in Ficus altissima and their ecological characters[J]. Chn J Ecol, 22(2): 70-73. [谷海燕, 杨大荣, 张光明, 彭艳琼, 宋启示. 2003. 高 榕隐头果内寄生蜂种类及生态学特征初步观察 [J]. 生态学杂志, 22(2): 70-73.]

Guan JM, Peng YQ, Yang DR. 2007. Host sanctions in fig-fig wasp mutualism [J]. Biodivers Sci, 15(6): 626-532. [管俊明，彭艳琼，杨大 荣. 2007. 榕一蜂互惠关系中榕树对未传粉榕小蜂的惩罚效应 [J]. 生物多样性, 15(6): 626-532.]

Hawkins BA, Compton SG. 1992. African fig wasp communities undersaturation and latitudinal gradients in species richness[J]. J Anim Ecol, 61(2): 361-372.

Herre EA, Knowlton N, Mueller UG, Rehner SA. 1999. The evolution of mutualisms: exploring the paths between conflict and cooperation [J]. Trends Ecol Evol, 14(2): 49-53.

Janzen DH. 1979. How to be a fig [J]. Annu Rev Ecol Syst, 10: 13-51.

Jousselin E, Rasplus JY, Kjellberg F. 2001. Shift to mutualism in parasitic lineages of the fig/fig wasp Interaction [J]. Oikos, 94(2): 287-294.

Kerdelhué C, Rasplus JY. 1996a. Non-pollinating Afrotropical fig wasps affect the fig-pollinator mutualism in Ficus within the subgenus Sycomorus. Oikos, 75(1): 3-14.

Kerdelhué C, Rasplus JY. 1996b. The evolution of dioecy among Ficus (Moraceae): an alternative hypothesis involving non-pollinating fig wasp pressure on the fig-pollinator mutualism [J]. Oikos, 77(1): $163-166$.
Kerdelhué C, Rossi JP, Rasplus JY. 2000. Comparative community ecology studies on Old World figs and fig wasps [J]. Ecology, 81(10): 2832-2849.

Kjellberg F, Gouyon PH, Ibrahim M, Raymond M, Valdeyron G. 1987. The stability of the symbiosis between dioecious figs and their pollinators: a study of Ficus carica L. and Blastophaga psenes L. [J]. Evolution, 41(4): 693-704.

Nefdt RJC, Compton SG. 1996. Regulation of seed and pollinator production in the fig-fig wasp mutualism [J].J Anim Ecol, 65(2): 170-182.

Proffit M, Schatz B, Borges RM, Hossaert-Mckey M. 2007. Chemical mediation and niche partitioning in non-pollinating fig - wasp communities. J Anim Ecol, 76(2): 296-303.

Ramirez BW. 1974. Coevolution of Ficus and Agaonidae[J]. Ann Miss Bot Gard 61(3): 770-780

Sun BF, Wang RW, Hu Z. 2008. Diet segregation of fig wasps and the stability of fig-fig wasp mutualism. Biodivers Sci, 16(6): 525-532. [孙 宝发, 王瑞武, 胡忠. 2008. 榕小蜂食性分化与榕树-榕小蜂系统稳 定性[J].生物多样性, 16(6): 525-532.]

West SA, Herre EA. 1994. The ecology of the New World fig-parasitizing wasps Idarnes and implications for the evolution of the fig-pollinator mutualism [J]. Proc R Soc Lond B, 258(1351): 67-72.

Wiebes JT. 1979. Co-evolution of figs and their insect pollinators [J]. Annu Rev Ecol Syst, 10: 1-12.

Yang DR, Zhao TZ, Wang RW, Zhang GM, Song QS. 2001. Study on pollination ecology of fig wasp (Ceratosolen sp.) in the tropical rainforest of Xishuangbanna, China [J]. ZoolRes, 22(2): 125-130. [杨 大荣, 赵庭周, 王瑞武, 张光明, 宋启示. 2001. 西双版纳热带雨林 聚果榕小蜂传粉生态学研究 [J]. 动物学研究, 22(2): 125-130.]

Zhai SW, Yang DR, Peng YQ. 2008. Distribution Patterns of Galls with Different Fig Wasps in Syconium of Monoecious Ficus racemosa [J]. For Res, 21(2): 145-149. [翟树伟，杨大荣，彭艳琼. 2008. 聚果榕雄 花前期隐头果内小蜂㾉花分布格局的初步研究 $[\mathrm{J}]$. 林业科学研究, 21(2): 145-149.] 